\title{
Spectral Analysis of Nanodiamond-Berberine Complex Interaction with Living Cells for Nanoparticle Mediated Drug Delivery
}

\author{
Yu-Chung Lin ${ }^{1,2}$, Zhe-Rui Lin ${ }^{1}$, Lin-Wei Tsai ${ }^{1}$, Elena Perevedentseva ${ }^{1,3}$, Artashes Karmenyan $^{1}$, \\ and Chia-Liang Cheng ${ }^{1^{*}}$ \\ ${ }^{1}$ Department of Physics, National Dong Hwa University, Hualien, Taiwan \\ ${ }^{2}$ Institute of Physics, Academia Sinica, Taipei, Taiwan \\ ${ }^{3}$ P. N. Lebedev Physics Institute of Russian Academy of Science, Moscow, Russia \\ * e-mail: clcheng@gms.ndhu.edu.tw
}

\begin{abstract}
The natural isoquinoline alkaloid berberine has been demonstrated for its significant activity against a variety of deceases caused by bacterial and parasite infections. Nanodiamond (ND) has also been proven to have great potential for drug delivery. In this work, the complex of ND with berberine is constructed through surface functionalized ND, aiming for drug delivery applications. Spectroscopic analysis was performed on the complex interaction with human lung alveolar carcinoma epithelial cell (A549) and red blood cell (RBC) in comparison with berberine in solution. The studied cells were treated with carboxylated ND, ND-berberine complex and berberine, respectively. Their distributions in cells structures are visualized using ND and berberine's fluorescence signals for detection. The distribution of ND and ND-berberine complex differs with the distribution of berberine introduced in solution form. The results show that the ND with attached drug molecules can act as a drug carrier allowing controllable localization of the drug into the cell. This result can have implication in drug delivery, release and delivery tracing applications. (C) 2017 Journal of Biomedical Photonics \& Engineering.
\end{abstract}

Keywords: Nanodiamond; Berberine; Bio-Applications; Medical Applications; Fluorescence; Surface Functionality; Imaging; Drug delivery; Nanobiotechnology.

Paper \#3162 received 27 Feb 2017; revised manuscript received 17 Apr 2017; accepted for publication 19 Apr 2017; published online 30 Apr 2017. doi: 10.18287/JBPE17.03.010305. [Special Issue. Years in Biophotonics: 70th Anniversary of Prof. A.V. Priezzhev].

\section{References}

1. D. Ho (ed.), Nanodiamonds: Applications in Biology and Nanoscale Medicine, Springer Science \& Business Media, New York (2009). ISBN: 978-1-4419-0530-7.

2. E. Perevedentseva, Y.-C. Lin, M. Jani, and C.-L. Cheng, "Biomedical Applications of Nanodiamond in Imaging and Therapy," Future Medicine. Nanomedicine 8(12), 2041-2060 (2013).

3. J. Walker, "Optical absorption and luminescence in diamond," Rep. Prog. Phys. 42, 1605-1659 (1979).

4. V. N. Mochalin, O. Shenderova, D. Ho, and Yu. Gogotsi, "The Properties and Applications of Nanodiamonds," Nat. Nanotechnol. 7, 11-23 (2012).

5. Y. Y. Hui, C. L. Cheng, and H. C. Chang, "Nanodiamonds for optical bioimaging," J. Phys. D Appl. Phys. 43(37), 374021 (2010).

6. A. Krueger, and D. Lang, "Functionality is key: recent progress in the surface modification of nanodiamond," Adv. Funct. Mater. 22(5), 890-906 (2012).

7. O. A. Shenderova, and G. E. McGuire, "Science and engineering of nanodiamond particle surfaces for biological applications (Review),” Biointerphases 10(3), 030802 (2015). 
8. E. Osawa, and D. Ho, "Nanodiamond and its application to drug delivery," J. Med. Allied Sci. 2(2), 31-40 (2012).

9. K.M. El-Say, "Nanodiamond as a drug delivery system: applications and prospective," J. Appl. Pharm. Sci. 01(06), 29-39 (2011).

10. R. Kaur, and I. Badea, "Nanodiamonds as novel nanomaterial for biomedical applications: drug delivery and imaging systems," Int. J. Nanomedicine 8, 203-220 (2013).

11. E. Perevedentseva, P.-J. Cai, Y.-C. Chiu, and C.-L. Cheng, "Characterizing protein activities of lysozyme and nanodiamond complex prepared for bio applications," Langmuir 27(3), 1085-1091 (2011).

12. Y. Kuo, T.-Y. Hsu, Y.-C. Wu, and H.-C. Chang, "Fluorescent nanodiamond as a probe for the intercellular transport of proteins in vivo," Biomaterials 34(33), 8352-836 (2013).

13. O. Faklaris, V. Joshi, T. Irinopoulou, P. Tauc, M. Sennour, H. Girard, C. Gesset, J.-C. Arnault, A. Thorel, J.-P. Boudou, P. A. Curmi, and F. Treussart, "Photoluminescent Diamond Nanoparticles for Cell Labeling: Study of the Uptake Mechanism in Mammalian Cells," ACS Nano, 3(12), 3955-3962 (2009).

14. K.-J. Huang, C.-Y. Lee, Y.-C. Lin, C.-Y. Lin, E. Perevedentseva, S.-F. Hung, and C.-L. Cheng, "Phagocytosis and immune response studies of Macrophage-Nanodiamond Interactions in vitro and in vivo," J. Biophotonics, Jan (2017).

15. Z. Chu, S. Zhang, B. Zhang, C. Zhang, C.-Y. Fang, I. Rehor, P. Cigler, H.-C. Chang, G. Lin, R. Liu, and Q. Li, "Unambiguous observation of shape effects on cellular fate of nanoparticles," Scientific Reports 4, 4495 (2014).

16. Y.-C. Lin, L.-W. Tsai, E. Perevedentseva, H.-H. Chang, C.-H. Lin, D.-S. Sun, A. Lugovtsov, A. Priezzhev, M. Jani, and C.-L. Cheng, "The influence of nanodiamond on the oxygenation states and micro rheological properties of human Red blood cells in vitro," J. Biomed. Optics 17(10), 101512 (2012)

17. L.-W. Tsai, Y.-C. Lin, E. Perevedentseva, A. Lugovtsov, A. Priezzhev, and C.-L. Cheng, "Nanodiamond for medical applications: interaction with blood in vitro and in vivo," Int. J. Mol. Sci. 17(7), 1111 (2016).

18. A. Alhaddad, C. Durieu, G. Dantelle, E. L. Cam, C. Malvy, F. Treussart, and J.-R. Bertrand, "Influence of the Internalization Pathway on the Efficacy of siRNA Delivery by Cationic Fluorescent Nanodiamonds in the Ewing Sarcoma Cell Model," PLoS ONE 7(12), e52207 (2012).

19. E. K. Chow, X.-Q. Zhang, M. Chen, R. Lam, E. Robinson, H. Huang, D. Schaffer, E. Osawa, A. Goga, and D. Ho, "Nanodiamond therapeutic delivery agents mediate enhanced chemoresistant tumor treatment," Sci. Transl. Med. 3(73), 73ra21 (2011).

20. X. Wang, X. Casuarine Low, W. Hou, L. N. Abdullah, T. B. Toh, M. M. A. Rashid, D. Ho, E. and K.-H. Chow, "Epirubicin-Adsorbed Nanodiamonds Kill Chemoresistant Hepatic Cancer Stem Cells," ACS Nano 8 (12), 12151-12166 (2014).

21. T. Schmeller, B. Latz-Brüning, and M.Wink, "Biochemical activities of berberine, palmatine and sanguinarine mediating chemical defence against microorganisms and herbivores," Phytochemistry 44(2), 257-266 (1997).

22. C. L. Kuo, C. W. Chi, T. and Y. Liu, "The anti-inflammatory potential of berberine in vitro and in vivo," Cancer Lett. 203(2), 127-137 (2004).

23. S. Jie, H. Li, Y. Tian, D. Guo, J. Zhu, S. Gao, and L. Jiang, "Berberine inhibits angiogenic potential of Hep G2 cell line through VEGF down-regulation in vitro," J. Gastroenterol. Hepatol. 26(1), 179-185 (2011).

24. M. Asai, N. Iwata, A. Yoshikawa, Y. Aizaki, S. Ishiura, T. C. Saido, and K. Maruyama., "Berberine alters the processing of Alzheimer's amyloid precursor protein to decrease Ab secretion," Biochem. Biophys. Res. Commun. 352(2), 498-502 (2007).

25. C. Caliceti, P. Franco, S. Spinozzi, A. Roda, and A. F. G. Cicero, "Berberine: New Insights from Pharmacological Aspects to Clinical Evidences in the Management of Metabolic Disorders," Curr. Med. Chem. 23(14), 1460-1476 (2016).

26. J. Yin, J. Ye, and W. Jia, "Effects and mechanisms of berberine in diabetes treatment," Acta Pharmaceutica Sinica B 2(4), 327-334 (2012).

27. S. Chen, X. Zhu, X. Lai, T. Xiao, A. Wen, and J. Zhang, "Combined cancer therapy with non-conventional drugs: all roads lead to AMPK," Mini Rev. Med. Chem. 14(8), 642-654 (2014).

28. W. S. Kim, Y. S. Lee, S. H. Cha, H. W. Jeong, S. S. Choe, M.-R. Lee, G. T. Oh, H.-S. Park, K.-U. Lee, M. D. Lane, and J. B. Kim, "Berberine improves lipid dysregulation in obesity by controlling central and peripheral AMPK activity,” Am. J. Physiol. Endocrinol. Metab. 296(4), E812-9 (2009).

29. S. S. Talwalkar, A. B. Vaidya, C. Godse, A. Vaidya, and R. Vaidya, "Plasmodium DNA Fluoresces With Berberine. A Novel Approach for Diagnosis of Malarial Parasites,” Am. J. Clin. Pathol. 124(3), 408-412 (2005).

30. Y.-J. Hu, Y. Liu, and X.-H. Xiao, "Investigation of the Interaction between Berberine and Human Serum Albumin,” Biomacromolecules 10(3), 517-521 (2009).

31. A. N. Colina, M. S. Díaz, and M. I. Gutiérrez, "Fluorescence of berberine in microheterogeneous systems," J. Luminescence 144, 198-202 (2013). 
32. S. Jantová, S. Letašiová, V. Brezová, L. Čipák, and J. Lábaj, "Photochemical and phototoxic activity of berberine on murine fibroblast NIH-3T3 and Ehrlich ascites carcinoma cells," J. Photochem. Photobiol. B: Biol. 85(3), 163-176 (2006).

33. P.-H. Chung, E. Perevedentseva, J.-S. Tu, C. C. Chang, and C.-L. Cheng, "Spectroscopic study of biofunctionalized nanodiamonds," Diam. Relat. Mater. 15(4-8), 622-625 (2006).

34. M. S. Diaz, M. L. Freile, and M. I. Gutierrez, "Solvent effect on the UV/Vis absorption and fluorescence spectroscopic properties of berberine," Photochem. Photobiol. Sci. 8(7), 970-974 (2009).

35. N. D. Strekal', I. G. Motevich, J. W. Nowicky, and S. A. Maskevich, "IR absorption and surface-enhanced Raman spectra of the isoquinoline alkaloid berberine," J. Appl. Spectr. 74(1), 31-37 (2007).

36. N. Bashmakova, S. Kutovyy, R. Zhurakivsky, D. Hovorun, and V. Yashchuk, "Vibrational spectra of berberine and their interpretation by means of DFT quantum-mechanical calculations," Ukr. J. Phys. 56(2) 130-137 (2011).

37. M. Megyesi, L. Biczók, and I. Jablonkai, "Highly Sensitive Fluorescence Response to Inclusion Complex Formation of Berberine Alkaloid with Cucurbit[7]uril," J. Phys. Chem. C 112(9), 3410-3416 (2008).

38. L. Xu, S. Hong, N. Sun, K. Wang, L. Zhou, L. Ji, and R. Pei, "Berberine as a novel light-up i-motif fluorescence ligand and its application in designing molecular logic systems," Chem. Commun. 52(1), 179-182 (2016).

39. M. Cao, M. Liu, C. Cao, Y. Xia, L. Bao, Y. Jin, S. Yang, and C. Zhu, “A simple fluorescence quenching method for berberine determination using water-soluble CdTe quantum dots as probes," Spectrochimica Acta Part A: Molecular and Biomolecular Spectroscopy 75(3), 1043-1046 (2010).

40. R. Reyes, G. Ramírez, and N. M. Delgado, "Fluorescent berberine binding as a marker of internal glycosaminoglycans sulfate in bovine oocytes and sperm cells," Arch. Androl. 50(5), 327-332 (2004)

41. W.-Y. Wu, J.-Y. Yang, L.-M. Du, H. Wu, and C.-F. Li, "Determination of ethambutol by a sensitive fluorescent probe," Spectrochim. Acta A Mol. Biomol. Spectrosc. 79(3), 418-422 (2011).

42. C.-Q. Zhou, J.-W. Yang, C. Dong, Y.-M. Wang, B. Sun, J.-X. Chen, Y.-S. Xu, and W.-H. Chen, "Highly selective, sensitive and fluorescent sensing of dimeric G-quadruplexes by a dimeric berberine," Org. Biomol. Chem 14(1), 191-197 (2016).

43. M. D. Crossfield, G. Davies, A. T. Collins, and E. C. Lightowlers, "The role of defect interactions in reducing the decay time of H3 luminescence in diamond," J. Phys. C: Solid State Phys. 7(10), 1909-1917 (1974).

44. Y.-C. Lin, K.-T. Wu, Z.-R. Lin, E. Perevedentseva, A. Karmenyan, M.-D. Lin, and C.-L. Cheng, "Nanodiamond for Biolabel and Toxicity Evaluation in the Zebrafish Embryo in vivo," J. Biophotonics 9(8), 827-836 (2016).

45. E. Perevedentseva, N. Melnik, C.-Y. Tsai, Y.-C. Lin, M. Kazaryan, and C.-L. Cheng, "Effect of surface adsorbed proteins on the photoluminescence of nanodiamond," J. Appl. Phys. 109(3), 034704 (2011).

46. W. Tan, Li, M. Chen, and Y. Wang, "Berberine hydrochloride: anticancer activity and nanoparticulate delivery system,” Int. J. Nanomedicine 6, 1773-1777 (2011).

47. Y.-H. Lin, J.-H. Lin, S.-C. Chou, S.-J. Chang, C.-C. Chung, Y.-S. Chen, and C.-H. Chang, "Berberine-loaded targeted nanoparticles as specific Helicobacter pylori eradication therapy: in vitro and in vivo study," Nanomedicine (Lond) 10(1), 57-71 (2015).

48. P. R. Vuddanda, A. Mishra, S. K. Singh, and S. Singh, "Development of polymeric nanoparticles with highly entrapped herbal hydrophilic drug using nanoprecipitation technique: an approach of quality by design," Pharm. Dev. Technol. 20(5), 579-587 (2015).

49. L. Wang, H. Li, S. Wang, R. Liu, Z. Wu, C. Wang, Y. Wang, and M. Chen, "Enhancing the Antitumor Activity of Berberine Hydrochloride by Solid Lipid Nanoparticle Encapsulation," AAPS PharmSciTech. 15(4), 834-844 (2014).

50. V. Vaijayanthimala, D. K. Lee, S. V. Kim, A. Yen, N. Tsai, D. Ho, H.-C. Chang, and O. Shenderova., "Nanodiamond-mediated drug delivery and imaging: challenges and opportunities," Expert. Opin. Drug. Deliv. 12(5), 735-749 (2014).

51. S Sreeja, and C. K. Nair, "Anticancer Property of Iron Oxide Nanoparticle-Drug Complexes: An In Vitro Study,” J. Environ. Pathol. Toxicol. Oncol. 34(3), 183-189 (2015).

52. M. Halimani, S. P. Chandran, S. Kashyap, V. M. Jadhav, B. L. V. Prasad, S. Hotha, and S. Maiti, "Dendritic effect of ligand-coated nanoparticles: enhanced apoptotic activity of silica-berberine nanoconjugates," Langmuir 25(4), 2339-2347 (2009).

53. M. Thakur, A. Mewada, S. Pandey, M. Bhori, K. Singh, M. Sharon, and M. Sharon, "Milk-derived multifluorescent graphene quantum dot-based cancer theranostic system," Materials Science and Engineering C 67 , 468-477 (2016).

54. D. Yu, P. Ruan, Z. Meng, and J. Zhou, “The Structure-Dependent Electric Release and Enhanced Oxidation of Drug in Graphene Oxide-Based Nanocarrier Loaded with Anticancer Herbal Drug Berberine," J. Pharm. Sci. 104, 2489-2500 (2015). 
55. B. R. Wood and D. McNaughton, "Micro-Raman characterization of high- and low-spin heme moieties within single living erythrocytes," Biopolymers 67(4-5), 259-262 (2002).

\section{Introduction}

Nanodiamond (ND) is recently considered to be a promising material for various bio-medical applications due to its unique features in sizes, structure, surface chemistry, physical properties and biocompatibility [1, 2] Particularly, the optical-spectroscopic properties of ND such as Raman and fluorescence [3, 4] open wide possible applications for bio-imaging and bio-sensing [5]. Its surface structure allows forming complex of ND with molecules of interests (drugs, antibodies, genes, etc.) $[6,7]$, and used as carrier for delivery of these molecules [8, 9] or the combination of delivery and imaging for monitoring and tracing [10].

Develop methods of delivery using ND implies analysis of functionality of bio-active molecules attached on ND [11, 12] and studies of mechanism of ND internalization by cells. It has been shown that nanodiamond of various sizes penetrates into different cells via clathrin-mediated endocytosis and localized in the cytoplasm [13, 14]. Nanodiamond's properties and surface modification with bio-active molecules can alter the ND penetration into cell [15]; this effect can be used to control the internalization of ND in cells. Functionality of proteins attached on ND (e.g. enzyme [11], hemoglobin [16, 17], lipoproteins [12], different drugs [8-10, 18-20]) has been investigated. Difference in mechanism of interaction of nanodiamond conjugates and pure drug with the target objects can modify the treatment [18], particularly, to overcome the chemoresistance of tumor $[19,20]$. Thus, using ND based conjugates allows improving efficiency of the treatment and decreasing the possible side effects.

It is obvious that optimal use of these advantages requires thorough studies of ND conjugates interaction mechanism with targeted systems. In the present work, we compare the internalization effects in cell of nanodiamond conjugate and pure drug. Nanodiamondberberine complex is prepared to study the interaction of nanodiamond-drug complex with cells using plant alkaloid berberine for the conjugation. Berberine, a clinically important natural isoquinoline alkaloid, has been extensively studied for its multiple biological activities, particularly its activity against bacteria, fungi and viruses [21]. A wide range of other pharmacological effects, including anti-inflammatory [22] and anticancer [23] effects, etc. [24] have also been reported. Recently the research interest on berberine is growing dramatically, large number of investigations show efficiency of the berberine in diabetes and metabolic syndrome treatments, improving glucose and lipid metabolism disorders $[25,26]$. Berberine targets a very basic regulator of metabolism adenosine monophosphate-activated protein kinase (AMPK), regulating energy homeostasis. Its action is based on increasing AMPK activity [27, 28].
Berberine exhibits well-detectable fluorescence, and some attempts to utilize this fluorescence were made, particularly for cellular imaging and diagnostics [29] and bio-sensing via florescence resonance energy transfer (FRET) [30]. Berberine fluorescence is sensitive to and can be modified by an environment chemical composition and properties [31]. It also has been reported as a photosensitive agent, which is able to produce singlet oxygen and radical species under UV irradiation [32]. The preparation of ND-berberine complex and analysis of delivery and release as well as chemo- and photosensitivity are interesting itself. In the present study berberine is used as a drug with bright fluorescence allowing its detection in the cell. In this study the red blood cells (human RBC) and human lung alveolar carcinoma epithelial (A549) cells are treated with ND-berberine complex, berberine in solution and ND. Their relative distributions in cellular structures are observed using ND and berberine's fluorescence signals for detection and analyzed to determine the difference from point of view of control of delivery.

\section{Methods and Materials}

\subsection{Nanodiamond and Nanodiamond- berberine complex preparation}

Nanodiamond and berberine complex was prepared using ND with nominal size $100 \mathrm{~nm}$ (Kay Diamond, US). Detail method of ND treatment has been reported elsewhere [33]. In short, nanodiamond was treated with mixture of strong acids $\mathrm{H}_{2} \mathrm{SO}_{4}: \mathrm{HNO}_{3}$ (1:3), to remove non-diamond admixtures and contaminations, and to modify the particles with $\mathrm{COOH}$ surface functional groups (carboxylated nanodiamond, cND) for further conjugation with desired molecules. Throughout the text, all the ND has been acid treated. For simplicity, in this work, ND means carboxylated nanodiamond.

Berberine (2, 3-methylenedioxy-9, 10dimethoxyprotoberberine Chloride) was obtained from Sigma-Aldrich (USA). To prepare the complex, $4 \mathrm{mg}$ of berberine powder was first dissolved in $1 \mathrm{ml}$ of standard phosphate buffer saline (PBS: $\mathrm{NaCl} 4 \mathrm{~g} ; \mathrm{KCl} 0.1 \mathrm{~g}$, $\mathrm{Na}_{2} \mathrm{HPO}_{4} 0.72 \mathrm{~g}, \mathrm{KH}_{2} \mathrm{PO}_{4} 0.21 \mathrm{~g}, \mathrm{H}_{2} \mathrm{O} 500 \mathrm{ml}$; pH 7.4) and heated to $40{ }^{\circ} \mathrm{C}$ for $30 \mathrm{~min}$ to increase solubility. Then, $2 \mathrm{mg} / \mathrm{ml}$ of ND was added to the berberine solution, obtaining its suspension with concentration of $2 \mathrm{mg} / \mathrm{ml}$. The mixture was thoroughly agitated for $2 \mathrm{hrs}$ for better adsorption of the berberine. After the agitation the mixture was centrifuged at the speed of 11,000 rcf at room temperature for 15 minutes to sediment the nanodiamond, including ND with adsorbed berberine. Then, $1 \mathrm{ml}$ of PBS solution ( $\mathrm{pH}$ value is 7.4) was added into the sediment, containing ND-berberine complex. The complex was subjected to weak ultrasound 
treatment to disaggregate the sediment and then to vortex for 30 minutes. The washing of ND-berberine complex from solution to remove non-interacting berberine was repeated 3 times.

\subsection{UV-Visible absorption measurements}

The absorption spectra of berberine solution before and after interaction with ND were measured using UVVisible spectrometer JASCO V-550 (JASCO, US) at room temperature. The intensity of adsorption peaks of the berberine are proportional to concentrations of the berberine in the solution, so comparison of the spectra allows rough estimation of quantity of adsorbed berberine and strength of the adsorption. In short, the spectrum of the prepared solution was measured to obtain the initial concentration of the berberine. Then, ND is added to adsorb the berberine. After centrifugation, the supernatant is measured. The difference the berberine adsorbed on the ND. The sediment is then washed with deionized water and centrifuged to ensure the sediment is ND-berberine, not ND and berberine mixture. The progress is repeated until we have zero absorption on the measured spectrum on the supernatant.

\subsection{Size and $\zeta$-potential measurements}

The particle size and $\zeta$-potential were analyzed using the Zetasizer Nano ZS (Malvern Instruments, Malvern, UK) with a $4 \mathrm{~mW}, 633 \mathrm{~nm}$ wavelength He-Ne laser on the base of dynamic light scattering method, with the detection angle $173^{\circ}$. Nanodiamond, berberine and NDberberine complex were diluted with PBS to measure size and surface charge to obtain the concentrations. After dilution to $20 \mu \mathrm{g} / \mathrm{ml}$, the $\mathrm{pH}$ was 7.4 at $25{ }^{\circ} \mathrm{C}$. The $\mathrm{pH}$ values were measured using a SENTRON $\mathrm{pH}$-meter (Titan, Taiwan).

\subsection{FTIR, Fluorescence and Raman spectra measurements}

For FTIR, fluorescence and Raman spectroscopic characterization, $20 \mu \mathrm{l}$ of ND, berberine or NDberberine complex solution each was dropped on a $\mathrm{Si}$ substrate $(1 \mathrm{~cm} \times 1 \mathrm{~cm})$ and dried in air under room temperature. FTIR spectroscopy (ABB Bomem MB 154 FTIR spectrometer, Switzerland) with a Deuterated TriGlycine Sulfate (DTGS) detector was used to obtain the sample's infrared spectra to confirm the forming of ND, berberine and ND-berberine complex respectively at temperature $25^{\circ} \mathrm{C}$ in air. The spectral resolution was 4 $\mathrm{cm}^{-1}$. The Raman and fluorescence spectra were measured using Raman spectrometer (T64000, Jobin Yvon, France) with $633 \mathrm{~nm}$ (for Raman spectra) and $325 \mathrm{~nm}$ (for fluorescence spectra) excitation wavelength.

\subsection{Red Blood Cells (RBC) solution preparation}

Fresh human blood was obtained from healthy volunteers and was transferred into the EDTA-covered tube; the research methods were approved by the Research Ethics and Use Committee of Buddhist Tzu Chi General Hospital (approval REC No. IRB101-149). The RBC were separated from the blood using centrifugation with the speed $1500 \mathrm{rcf}$ for $5 \mathrm{~min}$ at $4{ }^{\circ} \mathrm{C}$ (HERMLE Z $323 \mathrm{~K}$, Germany) and then repeatedly (5 times) washed with standard PBS by dissolving in ratio 1:2 and centrifugation with speed $3400 \mathrm{rcf}$ at $4{ }^{\circ} \mathrm{C}$ for 1 minute. Finally, the washed RBC was diluted with PBS in the ratio of $5 \mu \mathrm{l}: 1000 \mu \mathrm{l}$ (RBC: PBS).

To study the interaction of $100 \mathrm{~nm} \mathrm{ND}$, berberine and ND-berberine solutions with RBC, ND and NDberberine were suspended in PBS with concentration 10 $\mathrm{mg} / \mathrm{ml} .20 \mu \mathrm{l}$ of the ND+PBS suspension was mixed with $1500 \mu \mathrm{l}$ of PBS and added to $1005 \mu \mathrm{l}$ of RBC+PBS suspension. Final concentration of ND or ND-berberine in the prepared ND (or ND-berberine) +RBC+PBS sample used for the measurements was $\sim 20 \mu \mathrm{g} / \mathrm{ml}$. For the berebrine interaction with $\mathrm{RBC}$, the berberine was dissolved in PBS in concentration of $4 \mathrm{mg} / \mathrm{ml}$. The berberine solution $(100 \mu \mathrm{l})$ was mixed with $1980 \mu \mathrm{l}$ of PBS and added to $10 \mu \mathrm{l}$ of RBC suspension. The final concentration of berberine in the prepared berberine $+\mathrm{RBC}+\mathrm{PBS}$ sample used for the measurements was $\sim 200 \mu \mathrm{g} / \mathrm{ml}$.

\subsection{A549 cell culturing}

Human lung alveolar carcinoma epithelial cell A549 was obtained from Bioresource Collection and Research Center (BCRC) in Taiwan. A549 cells were cultured in RPMI1640 medium (Gibco, Invitrogen, UK). The medium was supplemented with $2 \mathrm{mM}$ L-glutamine (Invitrogen, USA), $1.5 \mathrm{~g} / \mathrm{L}$ sodium bicarbonate (Sigma, UK), 10\% fetal bovine serum (Gibco/Life Technologies, Carlsbad, CA, USA). Cells were maintained under standard cell culture conditions in an incubator (Galaxy 170S, Eppendorf, USA) containing 95\% air and 5\% $\mathrm{CO}_{2}$ at $37{ }^{\circ} \mathrm{C}$ humid environment. Culture medium was replaced with fresh medium every 48 or $72 \mathrm{hr}$. Cells were detached by treatment with $0.5 \%$ trypsin and 2.6 $\mathrm{mM}$ ethyl-enediaminetetraacetic acid (EDTA) (Gibco/Life Technologies, Carlsbad, CA, USA); cultures were subcultured routinely at approximately $80 \%$ confluence.

The A549 was treated with ND, berberine and NDberberine to observe their interaction. Each sample was added to the medium, the sample concentration in the medium was $20 \mu \mathrm{g} / \mathrm{ml}$, and cells were incubated together with the samples for 2 hours. Unreacted samples were removed by washing. The cells with ND, berberine and ND-berebrine adhered on the coverslips were used for microscopic investigations. 


\subsection{Cells Raman measurements and confocal fluorescence imaging}

Raman spectroscopic analysis of RBC was performed according to methods described previously $[16,17]$. For Raman spectroscopic investigation of ND-berberine and berberine interaction with RBC, the Raman spectra of $\mathrm{RBC}$ determined by hemoglobin $(\mathrm{Hb})$ were measured using a Raman spectrometer (T64000, Jobin Yvon, France) with $633 \mathrm{~nm}$ excitation wavelength laser. The prepared $\mathrm{RBC}+\mathrm{PBS}$ and the ND-berberine $+\mathrm{RBC}$ suspensions were placed in a Petri dish of $5 \mathrm{~cm}$ diameter; the Si substrate $2 \times 2 \mathrm{~cm}^{2}$ was kept at the bottom during the initial microscopic measurements to avoid the interference of the signals from the plastic Petri dish. The oxygenation/deoxygenation of NDberberine interacted $\mathrm{RBC}$ can be observed via the changes of a single RBC's Raman spectra. Oxygenation was achieved in ambient atmospheric pressure; deoxygenation was performed with nitrogen gas (10 $\mathrm{kg} / \mathrm{cm}^{2}$ ) purged into the Petri dish. RBC spectra were measured for one cycle of deoxygenation-oxygenation for a single $\mathrm{RBC}$, and each experiment was repeated with 5-10 RBCs.

Imaging of the cells (RBC and A549) after interaction with berberine, ND and ND-berberine complex was performed using a Leica laser-scanning confocal fluorescence microscope (TCS SP5, Mannheim, Germany) with $40 \times 1.25$ oil immersion objective (Leica, Mannheim, Germany). To localize ND and berberine relatively the cell structures the confocal scan along vertical direction (Z-scan) was applied. The berberine fluorescence was excited with $405 \mathrm{~nm}$ wavelength laser and signal was collected in the 530$620 \mathrm{~nm}$ wavelength spectral range; ND's fluorescence was excited with $488 \mathrm{~nm}$ wavelength laser and signal was collected in the 500-530 $\mathrm{nm}$ wavelength range.

\section{Results and Discussion}

The characteristic UV-Visible absorption spectra of berberine are shown in Fig. 1. The absorption spectrum reveals three strong bands with maxima around 250 and $350 \mathrm{~nm}$, which are characterized as $\pi \rightarrow \pi^{*}$ transition [34], and forth relatively weak band centered at about $430 \mathrm{~nm}$. In Fig. 1, spectrum (I) corresponds to absorption of the initial solution of the berberine prepared. Spectrum (II) is the supernatant after first centrifugation. The difference in the solutions spectra intensities is proportional to quantity of the substance (in this case berberine) which was removed from the solution in the result of the interaction with nanodiamond [33]. Spectra (III)-(VI) display spectra of supernatant after following washings of nanodiamond sediment. One can see that significant quantity of the berberine is washed out from the nanodiamond, especially at 2 nd washing (spectrum III). However the measurements discussed below show that the quantity of the berberine in ND-berberine complex is enough for observation and for further study of the complex interaction with cells.
The measured Raman and FTIR spectra of the berberine are presented in Fig. 2 after baseline correction. The vibrational spectra of berberine and some derivatives have been investigated previously using the Raman (including SERS) and IR absorption spectroscopy methods [35-36]. Both, Raman and FTIR spectra are complicate and the most intense peaks are positioned in the range $1200-1700 \mathrm{~cm}^{-1}$. The vibrational spectrum of berberine reveals $\mathrm{CH}$ bending and aromatic CC stretching modes, together with $\mathrm{CO}$ stretching vibrations, $\mathrm{CH}$ bending in the $\mathrm{OCH}_{2}$ and $\mathrm{OCH}_{3}$ groups, and aliphatic $\mathrm{CH}$ bending, corresponding to detailed attribution given in the literature [36]. Because the spectra are measured in ambient conditions, the $\mathrm{OH}$ vibrations around $1620-1650 \mathrm{~cm}^{-1}$ overlap with the sample spectra.

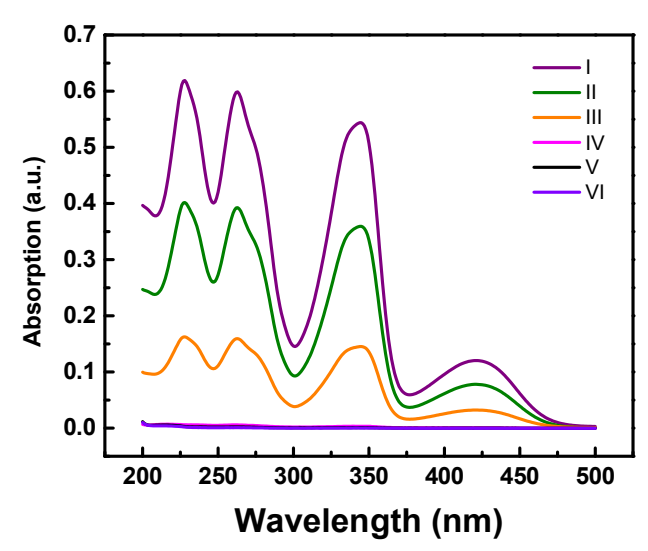

Fig. 1 UV-Visible spectra of berberine. (I) Initial solution of the berberine, (II) Supernatant after first centrifugation, (III)-(VI) Supernatant after following washing of nanodiamond sediment.

Raman spectrum of $100 \mathrm{~nm}$ ND reveals diamond peak of $\mathrm{sp}^{3}$ hybridized carbon at $1333 \mathrm{~cm}^{-1}$. The weak but observable diamond peak in the spectrum of NDberberine complex confirms the berberine adsorption on ND. While the agreement between ours, literature experimental data, and calculations [35-36] for the berberine Raman spectra are very good. However, for IR all measured spectra differ from calculated spectra significantly. This is because the dominant bands of the berberine are due to vibrations of highly polarized bonds and are therefore very sensitive to the experimental conditions (particularly, aggregation state and H-bonding) [35-36].

In Fig. 3, the sizes (a) and $\zeta$-potentials (b) of ND, berberine, ND-berberine complexes obtained using dynamic light scattering method measured at $\mathrm{pH} \sim 7.45$ are presented. The size of ND particles is close to nominal size $100 \mathrm{~nm}(155 \pm 20 \mathrm{~nm})$, so the degree of aggregation is low and well dispersed ND solution was achieved. In the case of ND-berberine complex significant fraction of non-aggregated particles (NDberberine complexes) with average size about $170 \mathrm{~nm}$ also exist. Surprisingly, the observed size of the berberine is of the same order, much larger than size 


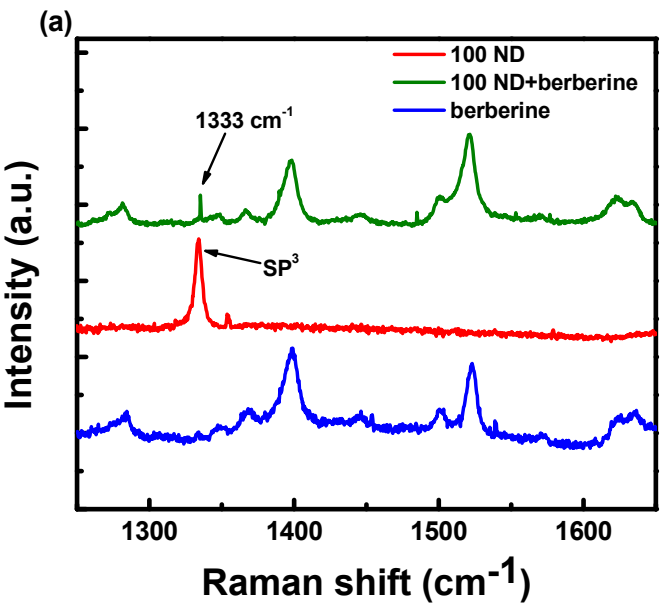

Fig. 2 Spectra of ND, berberine and ND-berberine complex: (a) Raman; (b) FTIR.

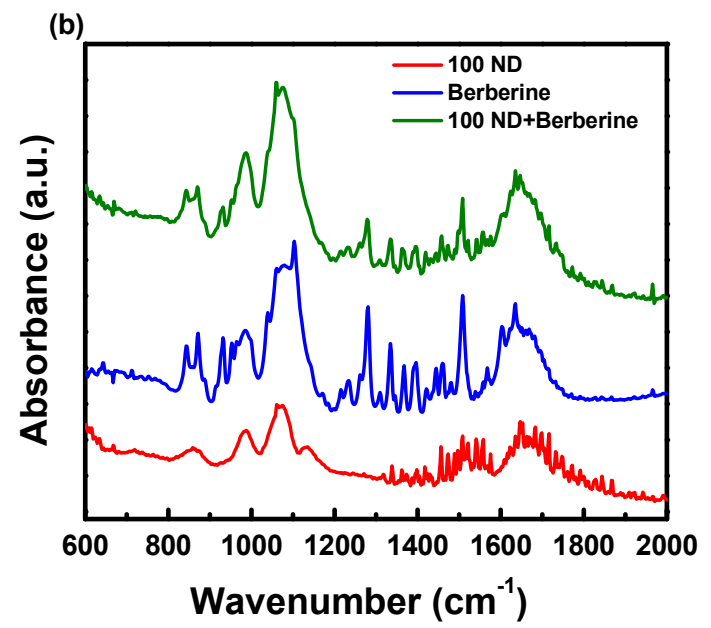

(a)

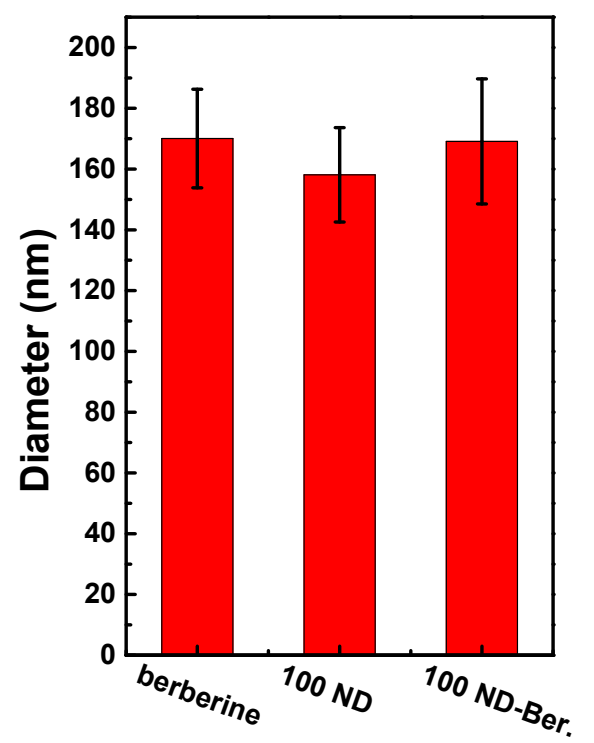

(b)

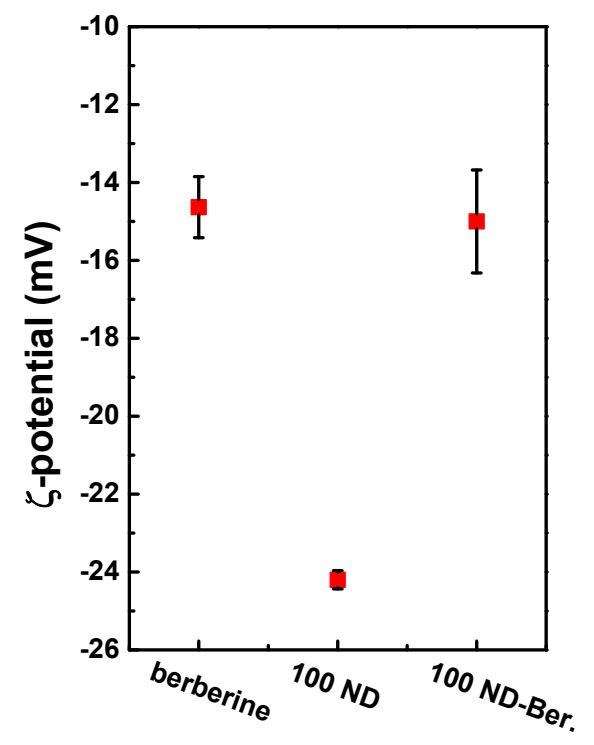

Fig. 3 The particles size (a) and zeta-potential (b) measured at pH 7.45.

of the berberine molecules. Berberine is known not very well soluble in water, so in this experiment we could have observed residual nanocrystals of the berberine, not molecules. The much larger size of the berberine can be a concern, but we follow our protocol to prepare ND complex with bio-molecules and didn't change it in this work except heating for comparison. We first prepare solution of the adsorbed molecules and then add ND. In this case we can estimate how much the biomolecules are adsorbed. High speed centrifuging does reduce the berberine size, but we will lose the information on the concentration. However, when NDberberine is formed, the complex size is within expectation, meaning the non-dissolved berberine did not participate in the reaction. The changes of $\zeta$ potential (Fig. 3b) from about $-24 \mathrm{mV}$ for $100 \mathrm{~nm} \mathrm{cND}$ to $-15 \pm 2 \mathrm{mV}$ for ND-berberine complex almost the same as the measured value for pure berberine, indicates the berberine modifies the ND surface to change the surface charges distribution. Judging from the size, NDberberine is formed with ND core and adsorbed berberine molecules (not crystal or cluster); while looking at the charge, ND-berberine forms overall charge similar that of clustering berberine also indicating we do have a layer of berberine on the ND. The complex charge of ND-berberine is determined by layers of berberine.

From Fig. 1, the intensity changes in the absorption spectra after the berberine adsorption on ND, and the measured size of the ND-berberine complex (in Fig. 3, close to ND) as well as the observed ND peak in Raman spectrum (Fig. 2b) allow confirming the formed NDberberine complexes are ND particles with adsorbed berberine molecules on the surfaces. Both ND and berberine have negative $\zeta$-potential; this can explain the 


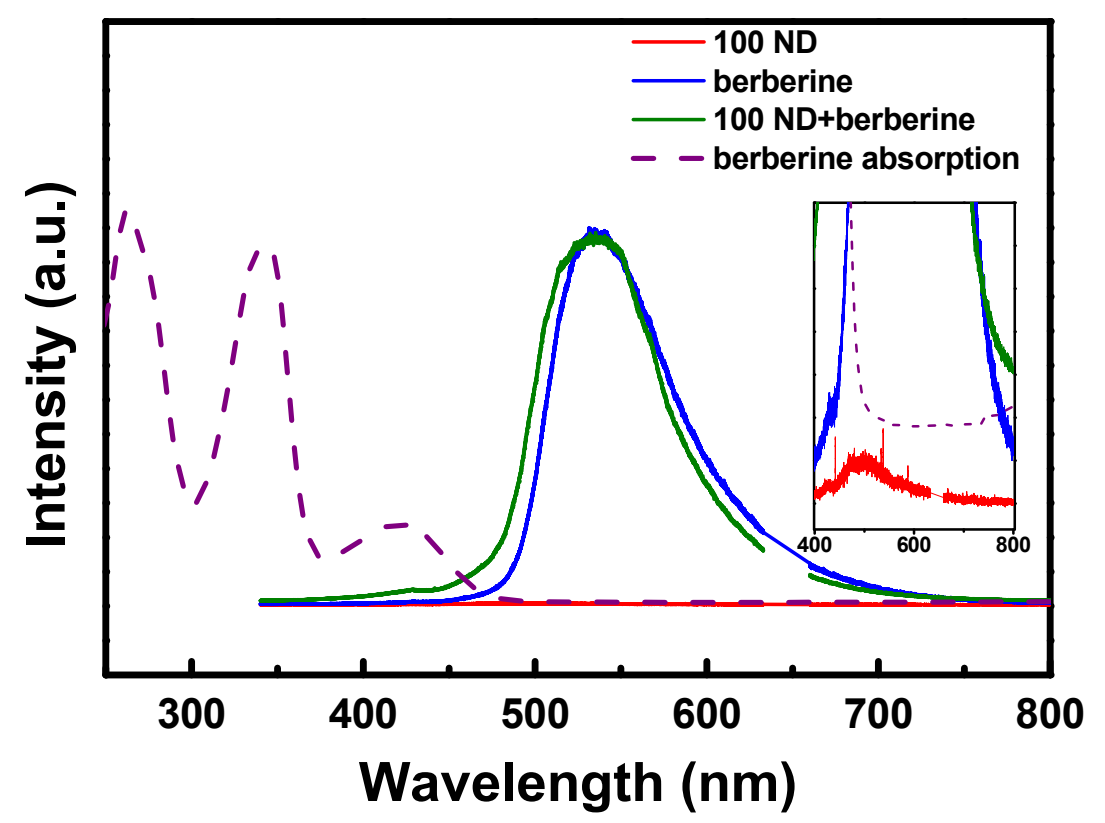

Fig. 4 Photoluminescence spectra of ND, berberine and ND-berberine complex (shown in solid lines) and spectrum of UV-Visible absorption of berberine (shown in dash line). Inset: extended spectrum of ND.

low stability of the complex in washing, observed via absorption spectra (Fig. 1).

Nanodiamond fluorescence is predominantly based on defect color centers in diamond structure. It has a number of advantages, such as stability, can be excited in wide range of excitations frequencies, etc. It is promising for imaging in bio-applications, including tracing of delivery of non-fluorescent substances (such as many drugs) [5]. In the present work we use highly fluorescent berberine, therefore we can simultaneously observe both the ND and berberine in the cellular model.

Photochemical properties of berberine have recently been investigated. The fluorescence of berberine can serve as fluorescent marker. In addition, both absorption and emission of berberine are sensitive to environment and can be used for sensing [34, 37-42]. Fluorescence spectra of berberine, ND, ND-berberine complex are shown in Fig. 4, together with absorption spectrum of berberine solution in PBS. The spectra were excited with $325 \mathrm{~nm}$ wavelength excitation laser where the energy is close to the berberine absorption maximum. The measured peak is intense, structureless and slightly asymmetric with a maximum near $535 \mathrm{~nm}$. Blue shift about $7 \mathrm{~nm}$ and slight change of the peak shape can be observed when berberine is adsorbed on ND surface. We can also observe (qualitatively) enhancement of adsorbed berberine fluorescence but can't estimate it quantitatively using our dried samples.

The intensity increasing and the shift of the fluorescence maximum of berberine when berberine interacts with environment have been described and analyzed previously, using non-polar solvents [34], DNA [37, 42] and many other bio-organic molecules and systems [e.g. 41]. The mechanisms are discussed, particularly, it was supposed that the interaction of berberine with these systems reduces the interaction with water (polar solvent) and hinders relaxation mechanism of the excited berberine [37]. Oppositely, effect of berberine derivative on quantum dot (QD) CdTe nanoparticle luminescence properties has been studied and it has been suggested that berberine adsorbing on the QD surface is responsible for disrupting electron-hole recombination process and resulting in the repression of luminescence [39].

The fluorescence image of 100 ND-berberine complexes is shown in Fig. 5. The fluorescence of 100 $\mathrm{nm} \mathrm{ND}$ is excited with $488 \mathrm{~nm}$ wavelength laser and collected in the 500-530 nm wavelength range (Fig. 5 (a)). The fluorescence of ND in this range corresponds predominantly to emission from diamond $\mathrm{H} 3$ defect centers with emission peak near $505 \mathrm{~nm}$ [43]. Berberine fluorescence was excited with $405 \mathrm{~nm}$ wavelength laser, corresponding to absorption band of the berberine. The signal was collected in the 530-620 nm spectral range; this region includes maximum and long-wavelength part of berberine fluorescence (Fig. 5 (b)). Fig 5c displays the merged (a) and (b) images, revealing the large (dried) aggregate of ND with adsorbed berberine. In addition, adsorption of macromolecules (such as berberine in this case) can slightly change the photoluminescence of the observed spectrum (Fig. 4), but does not affect the imaging [44, 45]. So, wide application of berberine in biophotonics can be expected due to its bright fluorescence, even in polar solvent like PBS, which can be used to observe the delivery into cell and localization of the drug inside in comparison with ND-drug complex.

Confocal images of A549 cell at their interaction with berberine and ND-berberine complex are presented in Fig. 6. In Fig. 6 (I), fluorescence images of A549 after $2 \mathrm{~h}$ incubation in berberine solution are presented. 

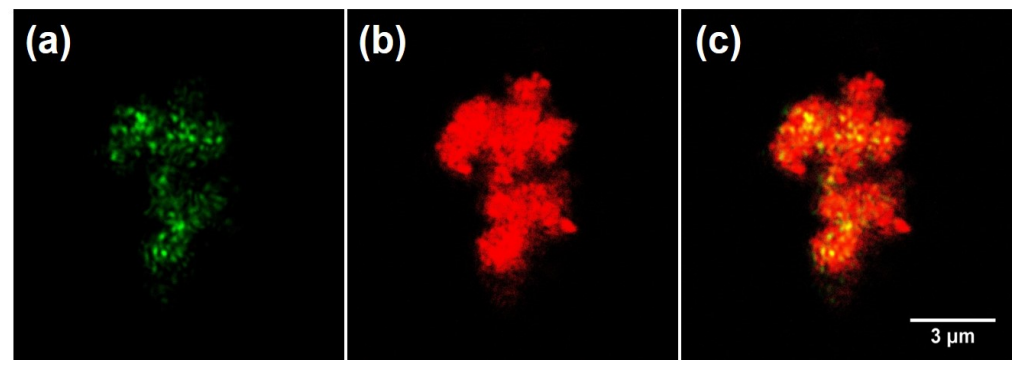

Fig. 5 Fluorescence image of aggregated 100 ND-Berberine complex: (a) $100 \mathrm{~nm}$ ND fluorescence excited with $488 \mathrm{~nm}$ wavelength laser, signal collected in the 500-530 nm range, shown in green color; (b) berberine fluorescence excited with $405 \mathrm{~nm}$ wavelength laser, signal collected in the 530-620 nm spectral range, shown in red color; (c) merged image of (a) and (b).
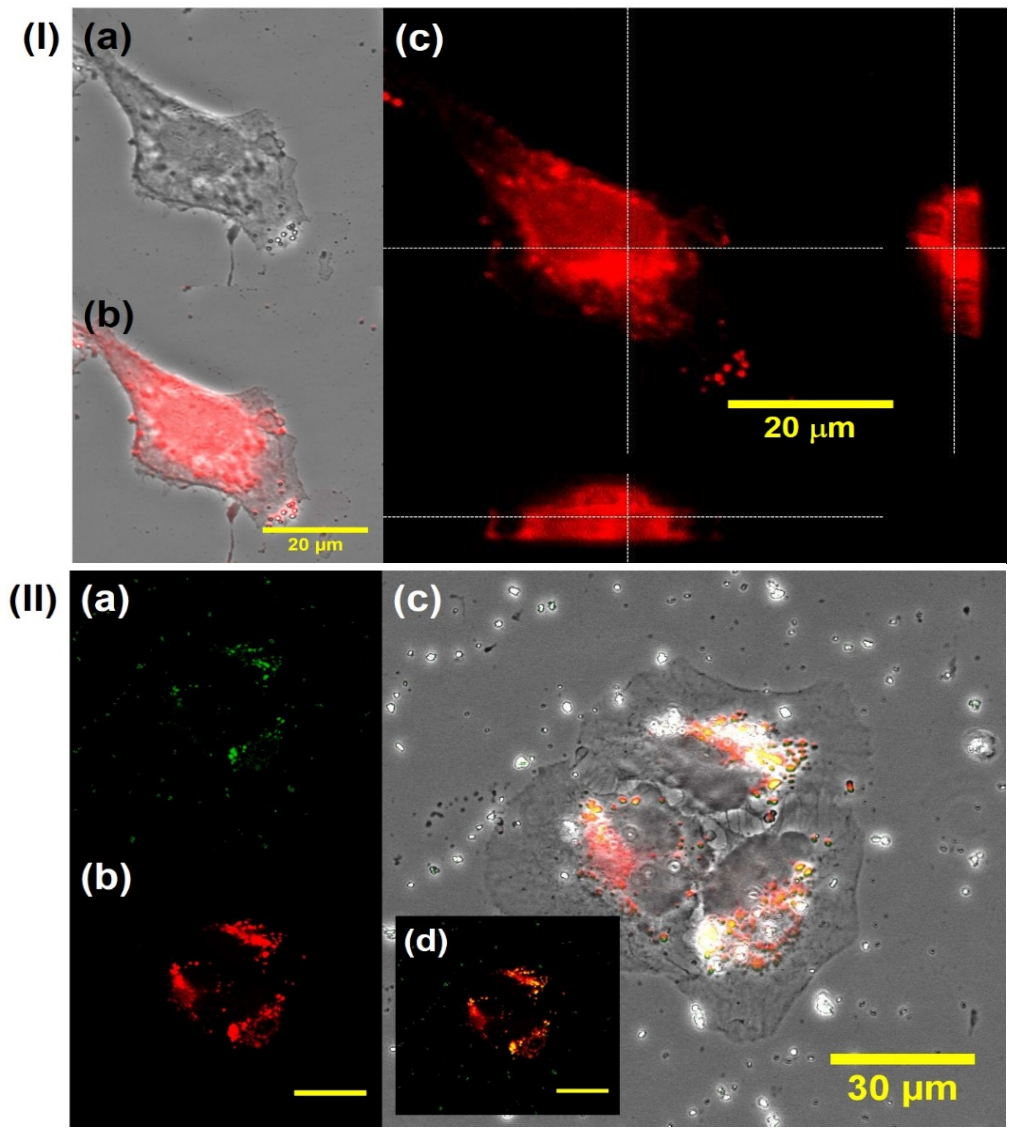

Fig. 6 Confocal imaging of A549 cell interaction with berberine and ND-berberine complex. (I) A549 with berberine: (I-a) Bright field optical image, (I-b) merged image of fluorescence and bright field optical images. (I-c) The x-z and yz projections of the z-scan of A549 with berberine. The z-scan is measured with a $250 \mathrm{~nm}$ step. (II) A549 with NDberberine complex. (II-a) Image of ND, fluorescence is excited with $488 \mathrm{~nm}$ wavelength laser and signal is collected in the 500-530 range; (II-b) image of berberine, fluorescence is excited with $405 \mathrm{~nm}$ wavelength laser and signal collected in the 530-620 nm spectral range. (II-c) Merged (II-a), (II-b) and bright field optical images. (II-d) is merged (II-a) and (II-b) fluorescence images.

Comparison of bright field optical image (I-a), fluorescence optical image (I-b) and analysis of the $\mathrm{z}$ scanning (I-c) allow observation the beberine's penetration inside and distribution in the cell. In Fig. 6(II) the images of A549 interacting with ND-berberine are shown. We also have compared these images with the cell images after incubation with $100 \mathrm{~nm}$ ND (not shown) which demonstrate observed previously distribution of ND in the cell cytoplasm [13, 14]. ND- berberine complex also is localized in the cytoplasm; the signals from ND (Fig. 6 (II-a)) and berberine (Fig. 6 (II-b)) are observed colocalized and overlapped (Fig. 6 (II-c)), so berberine is still not released from ND. While the observed distribution of ND and ND-berberine complex in the cell cytoplasm is similar after $2 \mathrm{~h}$ incubation (Fig. 6 (II)), berberine distribution in the cell is more homogeneous and it can gradually penetrate into nuclei (Fig. 6 (I)). Note that at longer incubation time 

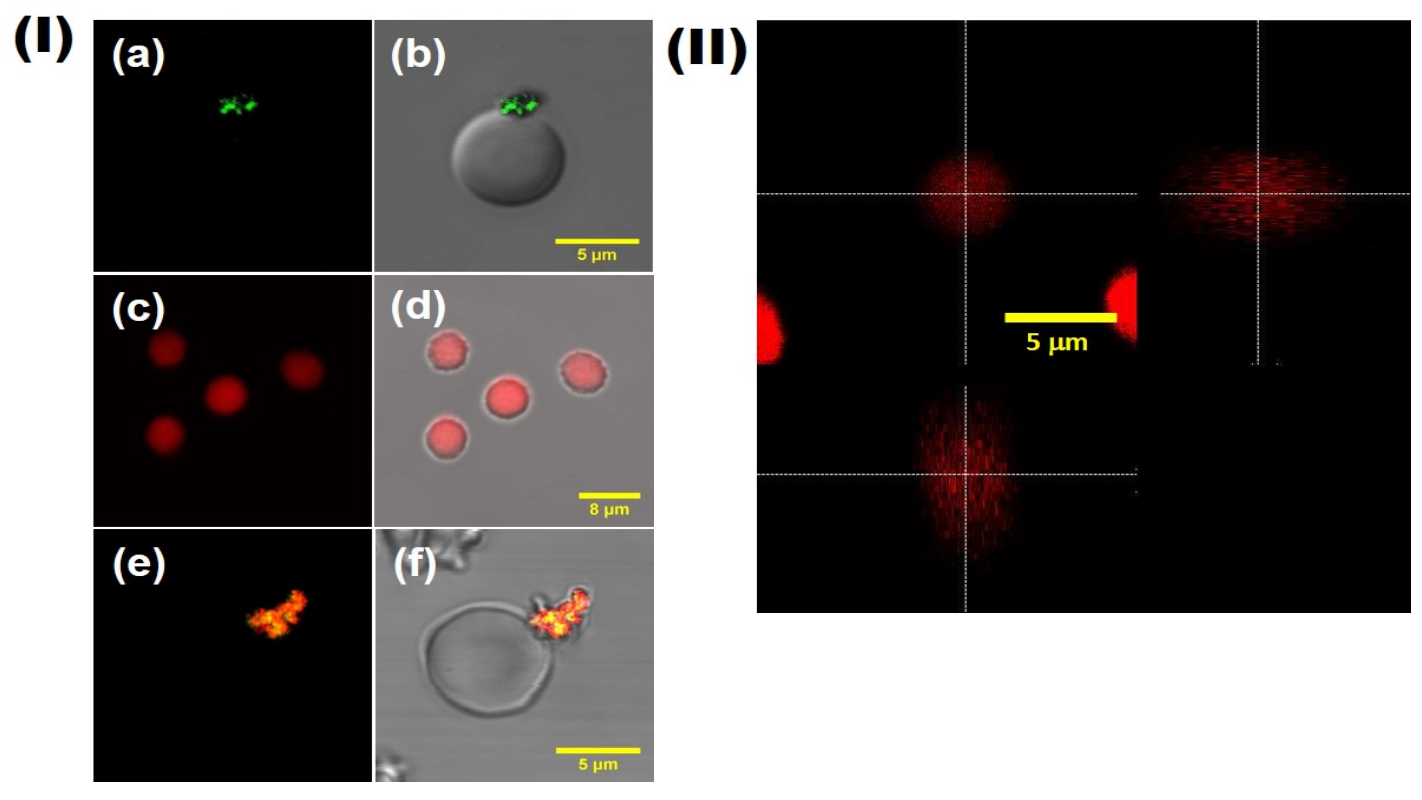

Fig. 7 (I) Fluorescence imaging of RBC interaction with 100 ND (a-b), berberine (c-d) and ND-berberine complex (ef). (II) The $x-z$ and $y-z$ projections of the $z$-scan of RBC with berberine. The $z$-scan is measured with a $400 \mathrm{~nm}$ step. The ND fluorescence is excited with $488 \mathrm{~nm}$ wavelength laser and signal is collected in the 500-530 nm range (shown in green color). The berberine fluorescence is excited with $405 \mathrm{~nm}$ wavelength laser and signal is collected in the 530$620 \mathrm{~nm}$ spectral range (shown in red).

(from $48 \mathrm{~h}$ ) berberine is colocolized with ND in less degree and can be observed in the nuclei area (not shown). But ND never penetrates into nuclei up to incubation of $48 \mathrm{~h}$ and more.

The works using nanoparticles (NP) containing or attaching berberine, first of all, are intended to overcome its low solubility, protect berberine from destruction by gastric acids, provide controllable release [46]. For that purpose chitosan porous particle [47], polymer [48] and lipid encapsulation [49] are usually used. Moreover, using nanoparticles, particularly ND, for targeted drug delivery expects increasing efficiency of the treatment $[2,50]$ utilizing various mechanisms. Among the discussed mechanisms [18-20], different pathways of the internalization by cells and controlled release are considered. Thus, Iron oxide $[50,51]$, gold [51], silica [52], graphene [53, 54] NP have been suggested as berberine carrier. The enhanced anticancer efficiency of $\mathrm{SiO}_{2}$-berberine complex has been observed [52]. It has been hypothesized that an increase in local concentration due to the confinement of a ligand on the nanosurface ("dendritic" effect) might have led to the observed effect. Unique properties of carbon-based NP, graphene and graphene oxide, are utilized to obtain new berberine carrier demonstrated as a system for anticancer drug delivery and fluorescent probe for multi-excitation [53]; graphene oxide electric and electrochemical properties were utilized for development of system allowing electric-sensitive drug release and enhanced oxidation of berberine as a model of metabolic regulation of drugs according to the different electrophysiological environment in tumor therapy [54]. These works show that potential of nanoparticles in the berberine applications and involving berberine investigations is high.

For further in-vivo applications, delivery of NDdrug complex to the targets via the blood flow will be a natural step. Therefore, the interaction of ND with the blood system is an important subject to study. In this work, in-vitro analysis of ND-berberine interaction with $\mathrm{RBC}$ was also performed. The microscopic fluorescence images of RBC after incubation for $30 \mathrm{~min}$ with ND, berberine and ND-berberine are presented in Fig. 7. In the Fig. 7 (I-a, b) ND fluorescence (shown in green) indicates the NDs are only attaching to the RBC cell membrane. The RBC incubated with berberine solution (in PBS solvent) is shown in Fig. 7 (I-c, d). The fluorescence is observed (shown in red) as a result of the berberine penetration into RBC. Fluorescence of berberine inside the RBC can be seen in the $x-z$ and $y-z$ projections of the z-scan (Fig. 7 (II)). For the NDberberine incubation with $\mathrm{RBC}$, its interaction with $\mathrm{RBC}$ is similar as for the ND. The images in Fig. 7 (I-e, f) show ND-berberine attached on the RBC membrane. Thus, we confirm the controllable localization of berberine in complex with ND on certain cellular structures.

We also have compared the effect of ND and NDberberine complex on RBC function using in situ Raman spectroscopic measurements; method has been presented previously $[16,17]$. RBC Raman spectra are determined by $\mathrm{Hb}$ inside and are very sensitive to the oxygenation and spin state dynamics of the heme (the active center of $\mathrm{Hb}$ ) due to a selective enhancement of the Raman active vibrations in different oxygenation states [55]. To check in a first approximation whether 
berberine adsorbed on ND's surface affects RBCs' function, Raman spectra of a single RBC incubated for 30 min with ND, berberine and ND-berberine have been measured at oxygenated and deoxygenated states (Fig. 8). The spectra of RBC in oxygenated state are shown in Fig $8 \mathrm{a}$, the RBC were oxygenated by air. Then, the same cells were deoxygenated by $\mathrm{N}_{2}$ purging the sample; the spectra are shown in the Fig. 8 (b). Finally, the cells were oxygenated again by air (Fig. 8c). In the process of deoxygenation and oxygenation the spectra exhibit significant changes. The major differences in the spectra are attributed to changes in the spin state of heme $\mathrm{Fe}$ and to the following conformational changes in $\mathrm{Hb}$ molecules [55]. The oxygenation degree of the samples can be monitored using the oxidation state marker bands, for example, $v_{4}$ in the range 1355 to 1380 $\mathrm{cm}^{-1}$, sensitive to the spin and coordination state of the heme, its changes are marked in the Fig. 8. Since RBC is relatively larger than berberine or ND-berberine, when it attaches to the RBC membrane, it did not affect the RBC oxygenation/de-oxygenation function. We didn't observe effect of berberine and ND-berberine complex on the RBC spectra both in oxygenated and deoxygenated state, as well as after repeated oxygenation. Thus, we can conclude that neither berberine, nor ND-berberine complex observably affect $\mathrm{RBC}$ state and ability to transfer oxygen. (a)

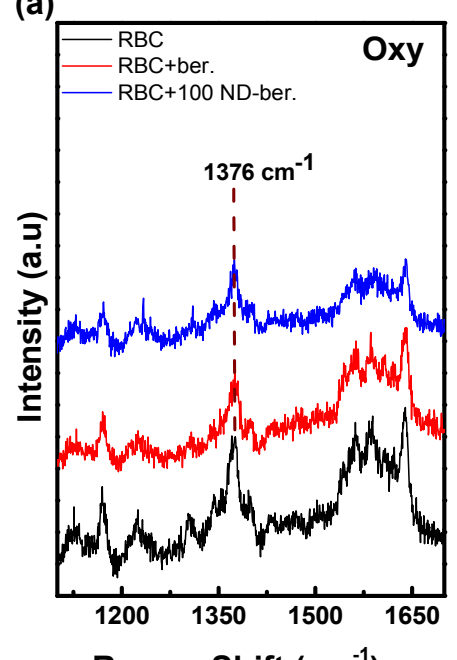

Raman Shift $\left(\mathrm{cm}^{-1}\right)$ (b)

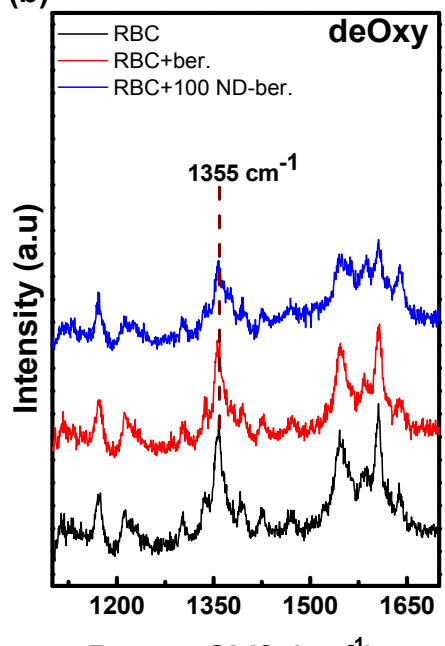

Raman Shift $\left(\mathrm{cm}^{-1}\right)$ (c)

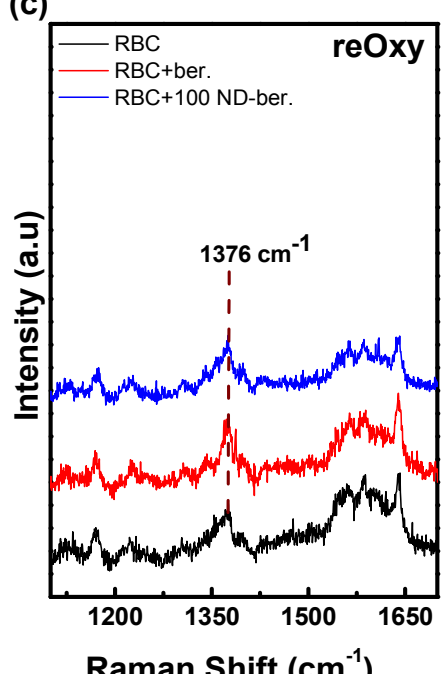

Fig. 8 Effect of berberine and ND-berberine complexes on Human Red Blood Cells: The Raman spectra of the RBC in oxygenated (a) deoxygenated (b) re-oxygenated states.

\section{Conclusion}

In this study the interaction of ND-berberine complex with human A549 cancer cell and human RBC are studied. The berberine was selected for its fluorescent properties, to observe simultaneously the nanodiamond and the berberine. The distribution of berberine, ND and ND-berberine relatively in cellular structures has been observed using independently ND and berberine fluorescence signals for detection. It is shown that NDberberine complex penetration into cell and the distribution of the berberine in the cell organells/structures is determined by ND. It allows controlling the berberine interaction with cell by attaching it on ND. We also show the interaction of ND, berberine, and ND-berberine doesn't affect the oxygenation properties of the human red blood cells. When connected to ND, the berberine distribution is determined by the ND, and attached to the cell membrane of RBC. This study also demonstrates the possibility of controlling the drug internalization in target cell which may increase the efficiency of drug treatment.

\section{Acknowledgment}

The authors appreciate the financial support of this research by the Ministry of Science and Technology (MoST) of Taiwan, Grant No. MOST 103-2112-M-259001-MY3; and for a Taiwan-Russia exchange program under Grant No. NSC 101-2923-M-259 -001 -MY3. 